\title{
THE PROCESS AND OPERATIONS OF SHAPE GENERATION AND MANIPULATION DURING THE ARCHITECTURAL DESIGNING ACTIVITY
}

\author{
Bouhelis, Walid; Arrouf, Abdelmalek \\ Laboratoire d'étude et de modélisation des phénomènes architecturaux et urbains, de l'idée à l'usage \\ (LEMPAU), University of Batna
}

\begin{abstract}
This piece of work is concerned with how shapes are generated, explored and transformed during the architectural designing process. It postulates that the relations and connections between sketches, produced during the design activity, can be defined in terms of shape transformations and described according to a closed list of shape operators. These latters provide a formal description of the shape exploration process and allow a deep understanding of its logic. To achieve its goal, this study creates a model to describe the different shape transformations, performed by designers, during the sketching activity.
\end{abstract}

Keywords: Early design phases, Shape operators, Design process, Protocol analysis, Design cognition

Contact:

Bouhelis, Walid

Laboratoire d'étude et de modélisation des phénomènes architecturaux et urbains, de l'idée à l'usage (LEMPAU), University of Batna-1

Architecture

Algeria

bouhelis_walid@hotmail.fr 


\section{INTRODUCTION}

Architectural design is now a privileged research subject, as shown by the number and the diversity of publications on this topic (Terzidis, 1989; Göel, 1995; Rodgers et al., 2000; De Biasi, 2000; Wetzel et al., 2006; Suwa et al., 2006; Arrouf, 2006; Lim et al., 2008; Prats et al., 2008; 2009). These works show that architectural design is a multi-phase process. One of them is the form manipulation and generation phase (Arrouf, 2006). During this incremental and iterative micro process, graphical simulation is frequently used. It allows, on one hand, to save ideas for future use and, on the other hand, to explore and to develop different design alternatives in a fast and flexible way. It involves the transformation of the overall form generated by designers or parts of it (Lim, 2008). Thus, the designer uses different types of representations, corresponding to different stages of the design process, to generate and evaluate possible responses to his design situation. He manipulates different formal configurations and moves from one conformation to another, using form transformation operations (Lim et al., 2008; Prats et al., 2009). Each sketch is transformed by the addition, deletion, modification or replacement of certain parts, until the point of satisfaction is reached and the answer he is looking for is definitively formulated (Prats et al., 2008, 2009). The main research question developed by this work is: how do designers generate shapes during the form manipulation and generation phase? Our investigation is particularly interested in the way that sketches are generated and manipulated, and the transformations of form that allow the deployment of such a process. The present work is based on an experimental study of the architectural design activity carried out by several designers. It aims to list all the shape transformations they mobilize, before formalizing them by means of a set of shape rules that explicitly describe the connections between sketches. This allows a better understanding of the drawing activity in the architectural designing process.

\section{FORM TRANSFORMATIONS AND SHAPE OPERATORS}

Several research projects were carried out in order to analyse and understand the different transformation processes and modification phases of the architectural design objects.

Göel's research (1995 in Prats et al., 2009) suggests that designers do not generate independent ideas but rather a single idea or a few related ideas and develop them through transformations. Göel identified two types of operations occurring between successive sketches during the different stages of design. These are lateral and vertical transformations (Göel, 1995). "Lateral or transversal" transformations convert one idea into a different idea, whereas "vertical" transformations is the movement from an idea toward a more detailed and precise version of it.

Wetzel (2009) suggests that the genesis of architectural forms results from successive transformations of shapes. He presents the concept of operator as a morphological action instrument. He argues that a design system, based on the combination of these operators, allows the designer to quickly explore a large number of spatial solutions. He claims that the process of creating architectural forms is based on the application of a set of morphological operators to initial forms (Wetzel et al., 2006). Operators such as: Bend, Twist, Crease, Wave, ... etc., have been identified and defined.

According to Prats (2009), the principles of the English language can be captured formally by the English grammar, through a set of linguistic rules. These latters are employed to compose new sentences that can be analysed and interpreted by others (Prats et al., 2009). Similarly, shape rules (Stiny and Gips, 1972 in Prats et al., 2009) can be used as a tool to describe shapes and shape transformations in a formal and visual way (Prats et al., 2009). As described by McCromack and Cagan (2002 in Trescak et al., 2012), a shape grammar is a method of generating designs. It is composed of shapes and shape rules. Using a shape grammar, designs are generated by starting with an initial shape and transformed by recursively applying these rules to a set of shapes (Trescak et al., 2012). They have been used as a means to analyse design objects in professions such as architecture and product design (Koning and Eizenberg, 1981; Agarwal and Cagan, 1998 in Trescak et al., 2012).

\section{CLASSIFICATION OF SHAPE OPERATORS}

Do et al. (2000) conducted a study on a set of drawings of a bungalow, designed by the architect Neimann. They attest that the relations between two drawings can be codified via a set of transformations applied to each element of the design object. They developed a classification of the 
elements and their different types of representation, angles of view, transformations, location and colours. The authors identified different shape transformations operated by the architect but they stopped at simple ones as rotation, translation or symmetry (wetzel et al., 2006).

In his book, Ching (2007) assumes that all forms can be understood as transformations of primary forms. These variations are generated by the manipulation of one dimension or more, by addition or subtraction of shapes or elements. He defined three types of transformations namely: dimensional, subtractive and additive transformations (Ching, 2007).

Wetzel (2006) suggests that the work of Francis D. K. Ching (2007) on architectural forms allows us to identify two main strategies of shape generation, and possibly two main classes of operators, known as the "transformation by composition" and the "transformation by metamorphosis", respectively represented by a lobster and a slug. These classes are related to the visual and compositional properties of the form defined by Ching (2007).

Based on a study of a collection of architectural and urban projects, represented in two dimensions, Borie et al. (2006) were able to classify the different modalities of shape deformation. The first class refers to the part of the shape on which the deformation is applied. It seeks to know the involved constitutive level of the composition. The second one deals with the way the element is distorted. In other words, it lists the possible types of deformations of an element or an architectural geometric system. Finally, the third class is interested in the extent of this deformation (Borie et al., 2006).

Another research conducted by Lim et al. (2008) states that shape transformations applied to successive sketches can be described via a limited number of operations. He classified these operations into two categories, namely: outline transformations and structural transformations. These were then broken down into several more specific formal operations.

A similar study realized by Prats et al. (2009) confirms the hypothesis, supported by Lim et al., that shape transformations in sketches can be used as a means to understand the design process. Indeed, in his paper, Prats et al. present an empirical study involving several freehand design activities, led by industrial designers and architects. This research suggests that the transformations of form applied to successive sketches can be described via a limited number of shape operations, in this case, seven. These are the following operations: outline transformations, transformations of the structure, substitution of an element, addition of an element, deletion of an element, division of an element, and finally change of view.

\section{DEFINITION OF SHAPE OPERATORS}

We note that, from this presentation of works on the transformation of the architectural form, the existence of a set of shape operators is the heart of many theories that support our own research. A transformation is defined here, as a process in which an object changes progressively its shape in order to obtain another one (Terzidis, 1989). It is the operation that modifies a real or a virtual form (Borie et al., 2006). These changes and variations are generated through the manipulation or alteration of one or more properties of the original form or element (Ching, 2007). The form for its part refers to both the internal structure and the external outline and the principle that gives unity to the whole (Ching, 2007). Stiny (1990 in Prats et al., 2009) defines it "as a finite arrangement of geometric elements, such as points, lines, and planes, each with a definite boundary and finite, but non-zero extent. Similarly, any arrangement of geometric elements that can be perceived to be embedded in a shape is defined as a sub-shape. Clearly any pictorial representation of a design, such as a sketch, can be formally represented as a shape, and any design elements that can be perceived to be a feature of the design can be formally represented as sub-shapes". Thus, the designer starts from an initial shape borrowed from various fields (geometry, nature, mechanics...) to a final shape capable of accommodating the owner's program. This path is made by numerous back and forth and important variations between different states of the shape, that makes it an iterative and parametric process. This process of creation of the architectural form is based on the application of a set of morphological operators to the initial forms (Wetzel et al., 2006). These operators are formalized according to shape rules, which explicitly describe the links between the sketches and allow a better understanding of the sketching activity in design (Prats et al., 2009). This work uses the concept of shape rules to explicitly describe shape transformations in sketches. In other words, it attempts to describe the path that designers have drawn in order to acquire an understanding of how shapes and elements are manipulated while exploring design alternatives. These shape rules reflect the types of shape transformations employed by 
designers (Jowers et al., 2010). "A shape rule is a rule of replacement of the shape A in B, where A and B are two shapes" (Prats et al., 2009). Prats et al. (2009) give three examples of an application of a shape rule that rotates a square $45^{\circ}$ around its centre, to shape $\mathrm{S}$. The shape rule is applicable to shape $\mathrm{S}$ because the shape which is located on the left side of the shape rule is a sub-shape of the shape S. Thus, the square, which is a sub-shape of the shape $\mathrm{S}$ can be rotated $45^{\circ}$ (Figure 1).

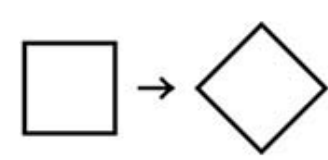

a

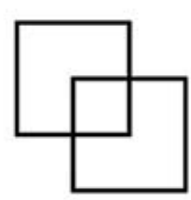

b
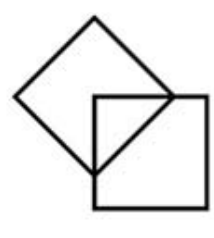

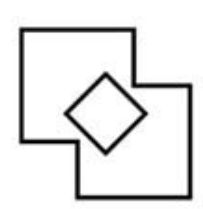

C

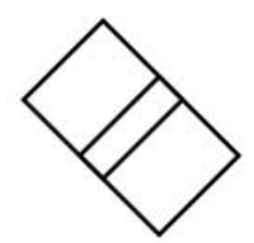

Figure1. a) A shape rule, b) a shape $S$, and c) examples of application of the shape rule to the shape S (Prats et al., 2009).

\section{PROTOCOL ANALYSIS METHOD}

One of the most used methods for capturing and collecting empirical data from design activity is the protocol analysis method. It is commonly used for the exploration of the design activity, because it allows conducting objective studies on the design process, to analyse its tasks and also to understand their progression in time (Arrouf, 2006). Design protocols take the general form of records of the designers' obvious behaviour, such as verbalization and sketching (Eckersley, 1988; Yang, 2002; Arrouf, 2006). Protocol analysis method is divided into two main approaches; the process-oriented approach and the content-oriented one (Arrouf, 2006; Suwa et al., 2006; Jiang \& Yen, 2009). The first focuses on the structural aspects of the design process. It is interested in the states of the problem, its operators, its procedures, its objectives and its strategies (Arrouf, 2006). While the content-oriented approach, adopted by this work, seeks to discover the types of information, resources and categories of knowledge manipulated by designers during the design process. Rather than discovering the underlying structure of the process (Arrouf, 2006 and Jiang \& Yen, 2009), it seeks to determine the cognitive and informational contents of the design activity (Arrouf, 2006). Two techniques of protocol analysis have been developed; think aloud and retrospective report techniques (Arrouf, 2006; Suwa et al., 2006). During the first technique, adopted by this study, the subjects are required to work on a given design task, while verbalizing their thoughts. The second technique invites the subjects to design first and then retrospectively report the earlier thoughts of their design processes. They use, for this, the visual aids provided by the video recordings, relating to their own design processes (Arrouf, 2006). The basic method of protocol analysis consists of the following sequence of tasks: elaboration of the protocols; description of the protocols and finally the treatment of the collected protocols (Eckersley, 1988; Yang, 2002; Arrouf, 2006; Suwa et al., 2006).

\subsection{Experimental design}

Our research adopts an experimental method that focuses on the observation of a design situation involving a freehand sketch activity by each participant. Three architects participate in our study. We refer to the three participating architects as Subject 1 to Subject 3. Subjects 2 and 3 are experimented architects because they have had various experiences in the field of architectural design for ten years at least. On the other hand, subject 1 is a novice architect because his experience does not exceed three years. We use for this paper, the data given by Subjects 1 and 2 in order to refer to a novice designer and to an experimented one.

\subsection{Design task}

The experiment takes place in two phases, for a total duration of fifty-five minutes. Each phase corresponds to a design task. The first task is a "warm-up exercise". It is ten minutes long. The second one is the actual experimental design. It lasts for forty-five minutes. During this phase, the subject is asked to design a house on an open site. He is invited to generate and to draw freehand sketches on 
paper and simultaneously produce an argumentative discourse. The design task performed by the participants is recorded on video. The following figure (Figure 2) illustrates one of the three subjects doing the design task we are discussing here.

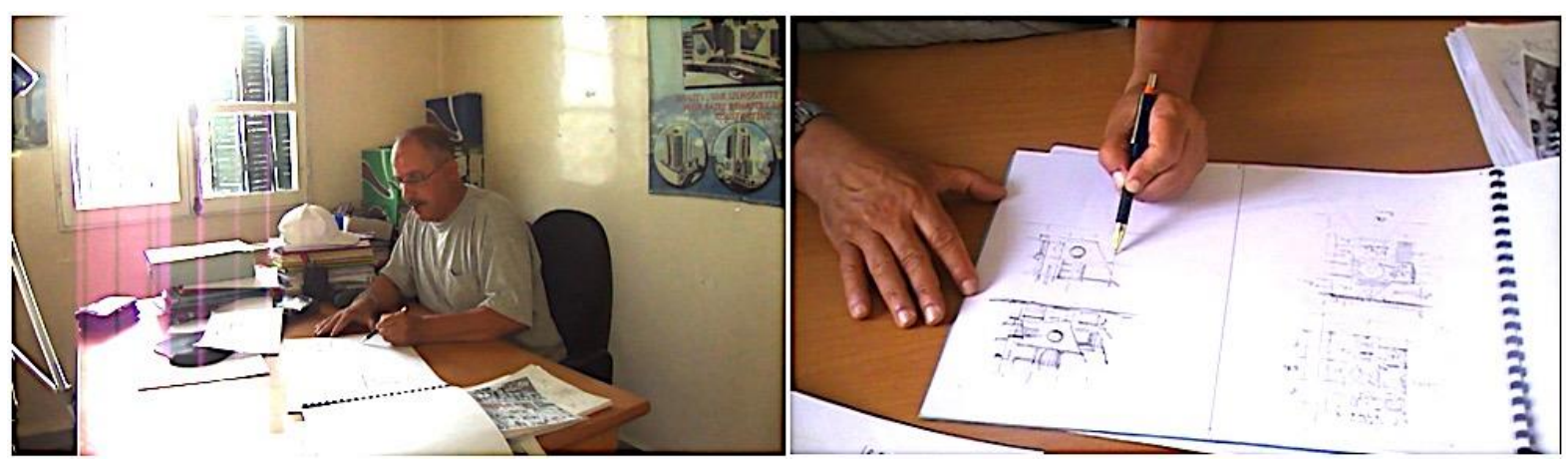

Figure 2. The design task as performed by the subject 2.

\subsection{Description of the protocols}

\subsubsection{Segmentation}

After the data collection, the raw protocol is divided into small units called "segments" (Arrouf, 2006). This work adopts the segmentation method developed by Suwa, Purcell and Gero (1998) and by (Arrouf, 2006). It stipulates that a single segment can contain multiple codes. These codes correspond to different shape operators carried out by designers in each segment, in this case the sketches produced by the subjects. Protocol analysis generally rejects graphical productions. All studies are limited to the verbal protocols and use graphical data as a simple complement for their understanding. This work considers, however, that graphical productions are carriers of information. They are crucial for any architectural design activity. It suggests that each produced sketch implies a thought around a given element / space / subject, this is why it contains an important informational value. Thus, during segmentation, our research considers each graphical element as a segment (Arrouf, 2006). This is all the more true as this investigation seeks to understand the logic of form manipulations and shape transformations that allow the deployment of such an activity, within the design process. This is why graphical representations are here essential and unavoidable. They form the support that allows us to find the different shape operators, used by architects during the design activity.

\subsubsection{Codification}

The coding of the segments represents the second step of the description of the protocols. It uses a coding model (coding scheme) that allows the exploitation of the protocols. It is a collection of groups of descriptors that serve as a medium for describing the design process, for its analysis (Arrouf, 2006). The coding scheme is developed according to the type of the study and the pre-established models of the design activity. To describe its protocols, this work adopts a coding strategy based on the model of shape operators, elaborated by our study and presented below. Once the sketches are classified according to their order of generation, they are grouped into tables of transformation, where they are compared two by two. Each sketch is compared with the one that immediately precedes it, according to the order of occurrence and according to the reference space. The transformations of form between consecutive sketches are identified, described and codified, through the predefined shape operators.

Figure 3 shows as an example, the shape operators used to codify the transformations observed between Sketch 7 and sketch 15, produced by subject 1. For example, operator 1: Geometrical relations operator : Rotate an envelope (living room), operator $2:$ Size operator : Extend the length of an envelope (Dining Room) and operator 3 : Conformation (shape) operator : Deviation of a frame (Hall). These operators describe very specific shape transformations. This is why numerous operators were necessary to describe all the shape transformations, mobilized by all the subjects, during their respective design tasks. Thirty two operators were, for example, necessary to describe all the shape transformations operated in sketch 15. 


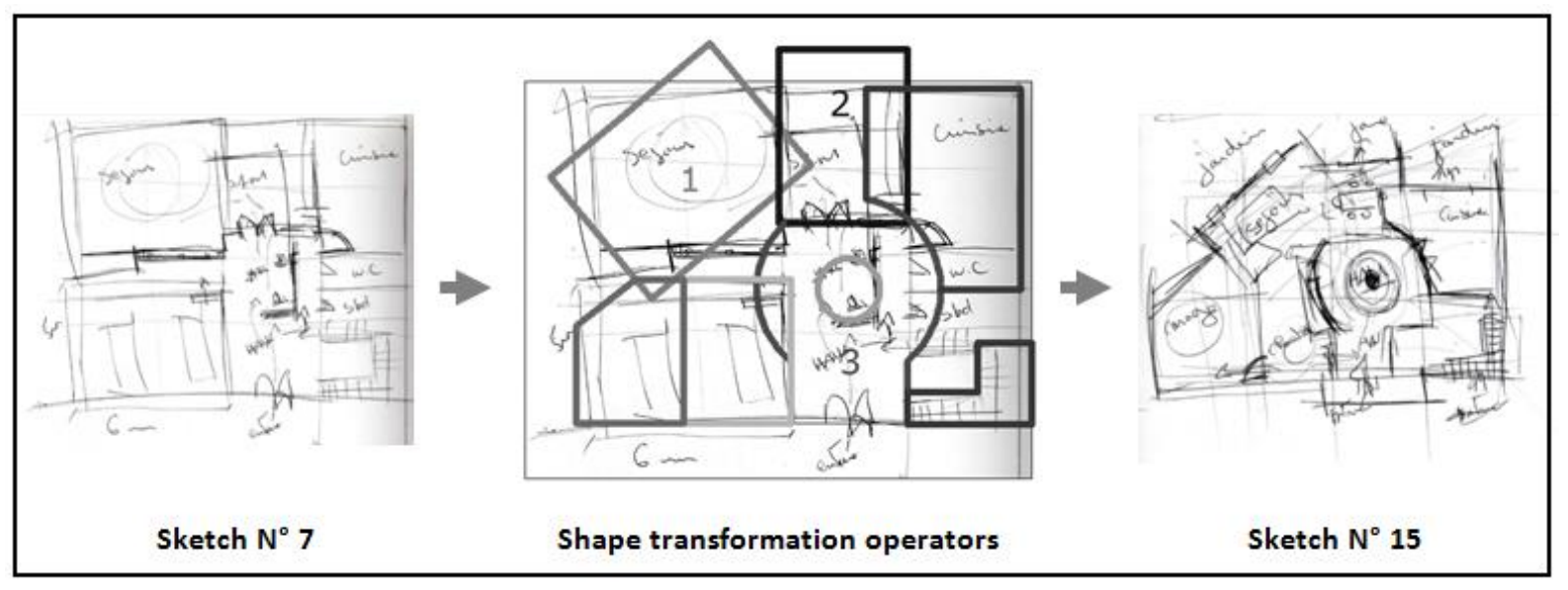

Figure 3. Examples of shape transformation operators.

\section{PRESENTATION OF THE SHAPE OPERATORS' MODEL}

The shape operators' model elaborated by this study, is based on the works that deal with how forms are generated and manipulated, and the transformation operations that allow the deployment of such a process. Based on the works developed by: Borie et al. (2006); wetzel et al. (2006, 2009); Ching (2007); Do et al., (2000); Lim et al. (2008); Prats et al. (2009); Rodgers et al. (2000) and De Biasi (2000), this work has defined a codification model that consists of three categories of shape operators, namely plastic shape operators, structural shape operators and figurative shape operators. These latters are decomposable into fourteen classes of shape operators (Table 1, categories and classes of the shape operators' model). Plastic shape operators and structural shape operators are identified and classified with regard to the properties of the form defined by Ching (2007), namely: the visual properties (plastic) and the compositional (structural) properties. The category of figurative shape operators is created, to qualify the transformation that does not relate to the shape of the design. It is about visualization and exploration.

\subsection{Plastic shape operators}

Plastic transformations correspond to the first strategy of Ching (2007) identified as a slug. We refer to it as "transformation by metamorphosis" (Wetzel et al., 2006) or "plastic transformation". The word "plastic" refers to the external appearance and to the outline of an element or a form, to the set of lines, shapes or colours. Plastic transformations are mainly used for the refinement of the architectural form (Prats et al., 2009). They consist of four classes of actions. These classes describe the transformations affecting the purely visual properties of a shape or an element, namely: conformation transformation (shape), size transformation, colour transformation, and texture transformation (Ching, 2007) (Table $1)$.

\subsection{Structural shape operators}

The work of Francis D. K. Ching (2007) on architectural forms also allows us to identify the second strategy that is metaphorically represented by a lobster. It consists of creating shapes through adjustment and combination. The word "structure" means the "internal constitution" or the "internal relations" of the architectural object; the relationships between shapes and elements, namely: geometrical relations and topological relations (Borie et al., 2006), we will refer to these transformations as "Structural transformations" or "transformations by composition" (Wetzel et al., 2006). Structural transformations are mainly used to express different spatial relationships between elements and to explore various solutions that relate to the organization, the layout and the constitution of the design. They are more likely to lead to radical changes in the shape than outline transformations (Prats et al., 2009). It consists of three classes of shape operators namely: topological relations transformation (position), geometrical relations transformation (orientation) and constitutive transformation (Borie et al., 2006; Ching, 2007). The table bellow shows the different classes of the Structural shape operators (Table 1). 


\subsection{Figurative shape operators}

Unlike the other categories of shape operators, defined above, the figurative shape operators do not represent transformations of the shape of the design. They are mainly used to allow the exploration and the visualization of the operations of transformation undertaken by designers. Their purpose is to test and detail graphically figured solutions, formulated by designers. This category describes the transformations affecting: the type of representation, the operators space / object, the operators space/ Floors (De Biasi, 2000), the point of view (Prats et al., 2009), the direction of view, the size of the sketch, and the level of complexity (Rodgers et al., 2000) (Table 1).

The transformations of form applied to successive sketches are described via a limited number of shape operators. While these operators, presented here, are sufficient to capture the transformations of form, made by the architects participating in our study, the set of the shape operators is not supposed to be complete. It is possible that other experiments may result in additions to the set. Nevertheless, there are three main categories of shape operations: plastic shape transformations, structural shape transformations and figurative shape transformations. Table 1 illustrates this set of shape operators, with illustrations in the form of shapes rules that express each operator in an abstract way.

Table 1. Categories and classes of the Shape transformation operators' model, elaborated by the present study.

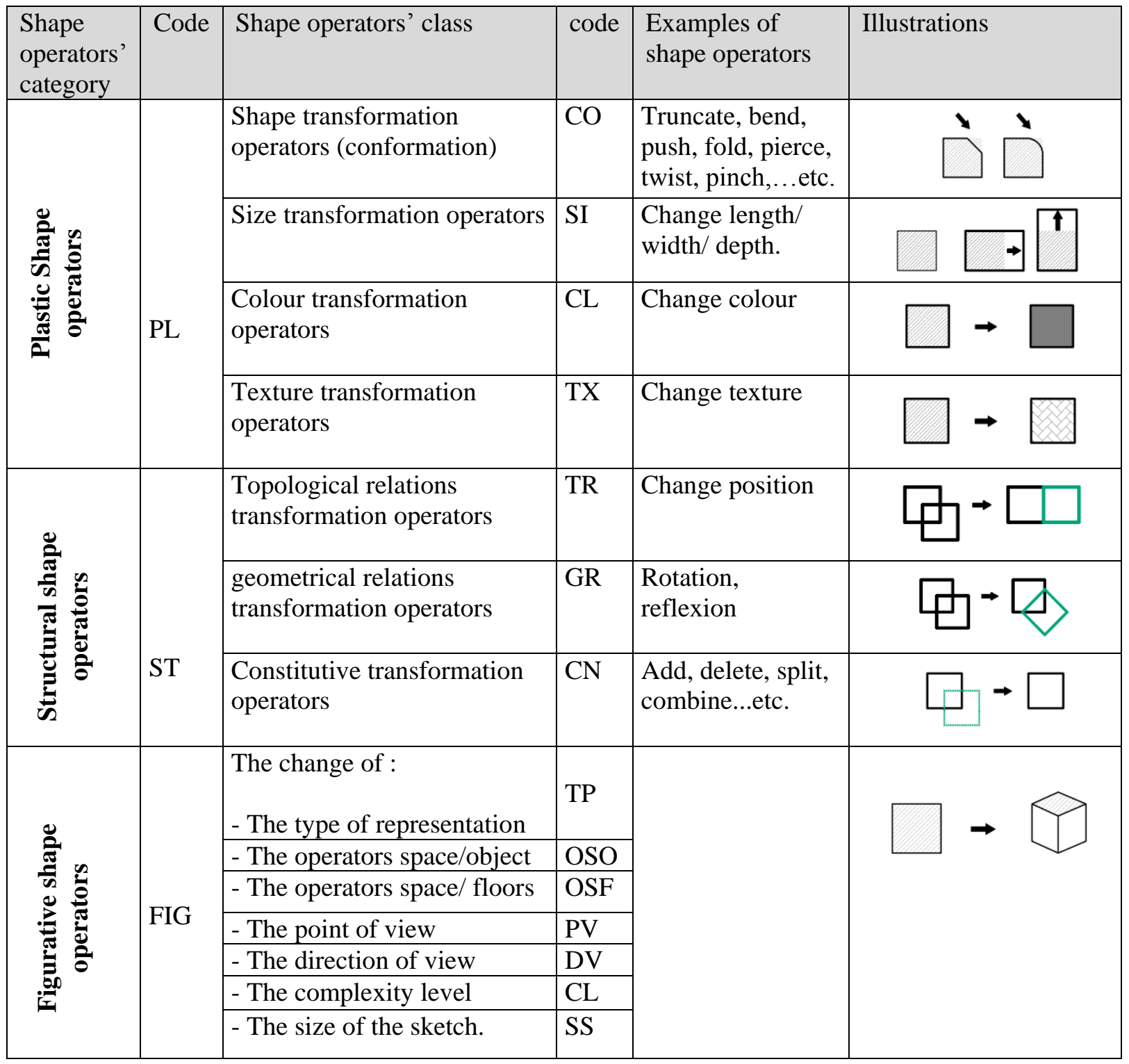




\section{TREATMENT OF THE DATA}

The sketches produced by each of the participating architects are classified in coding tables. Every sketch is considered as a separate segment and it has been the subject of a detailed description. The video recordings have been a great help as for identifying the order and the meaning of the different sketches, generated by the two designers, selected for this study. All the transformations of form made by the subjects, between consecutive sketches are identified, described and codified via the model of shape operators, elaborated by this work. The protocols are codified by the two successive codifications method, developed by Suwa, Gero and Purcell (1998). According to this method, a single coder codifies the protocols for the first time and then he redone a second coding as soon as the first is complete. The same coder arbitrates then the two coding passages to produce the final coding. (Arrouf, 2006)

\subsection{Quantification of the shape operators}

The coding of the segments is transcribed, for each subject in an "objects / attributes" table (Arrouf, 2012). Sketches are the objects of the table and the categories of shape operators are its attributes. Thus, the table records the number of occurrence of operators per sketch (Table 2).

Table 2. A part of the operators' quantification table of the protocols of subject 1.

\begin{tabular}{|l|c|c|c|}
\hline Operators categories & $\begin{array}{c}\text { Plastic operators } \\
\text { PL }\end{array}$ & $\begin{array}{c}\text { Structural operators } \\
\text { ST }\end{array}$ & $\begin{array}{c}\text { Figurative operators } \\
\text { FIG }\end{array}$ \\
\hline Sketch 2 & 0 & 5 & 0 \\
\hline Sketch 3 & 1 & 5 & 1 \\
\hline Sketch 4 & 0 & 1 & 1 \\
\hline
\end{tabular}

The number of specific shape operators refers to the previous chronological sketch and to the previous referential one. The referential sketch refers to the one that includes the state of the sub-shape or the element before its transformation.

\subsection{Validation and evaluation of the shape operators model}

This part of the analysis seeks to demonstrate that the transformations of form, operated by the subjects, can be described by means of the shape operators, previously identified and listed by the model, here created. To do this, it is carried out in two steps. The first validates the content of the model while the second confirms its structure.

\subsubsection{Evaluation of the content of the model}

The description and the codification led by this work shows that the operators, categories and classes of the shape operators model, have all been solicited to describe and to codify the shape transformations performed by the subjects. Besides, our model has supported all the operations of the subjects. This is why we can affirm that all the operators of the model of form transformation, elaborated by this study, are necessary to describe the activity of form manipulation and generation of the architectural design process, in an exhaustive and complete way.

\subsubsection{Evaluation of the structure of the model}

The model, developed by the present work, structures the operators of form production and manipulation, used by the participating subjects, during the design activity, in three categories, namely: plastic shape operators, structural shape operators and finally figurative shape operators' category.

This classification turns out to be appropriate. This is confirmed in particular by the factorial analysis of the data of the two architects. In fact, this analysis isolates each category of shape operators apart and opposes, via axis 2, the structural category to the plastic and figurative ones as shown in the results in Figure 4. 

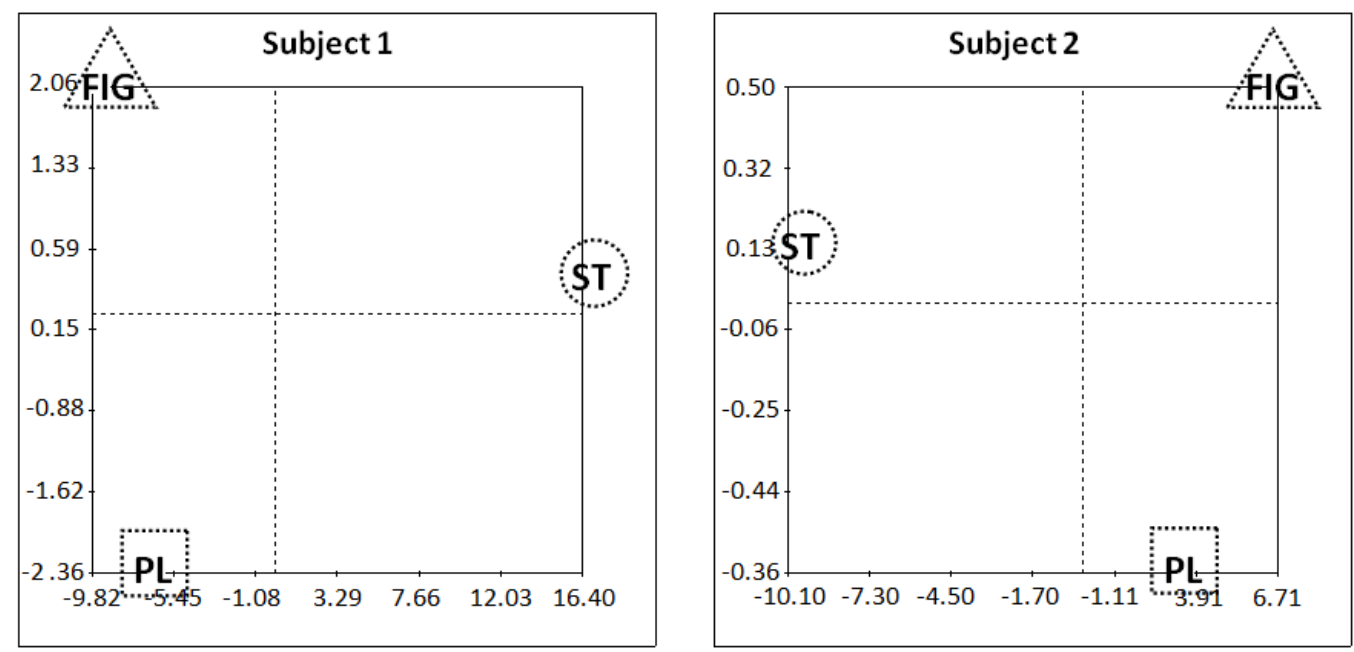

Figure 4. Results of the factorial analysis of the subject 1 and subject 2 protocols (PL: plastic, ST: structural, FIG: figurative).

This classification is also confirmed by the results of the cluster analysis, shown in the graphs below (Figure 5). The dendograms of the two subjects show that the shape operators are effectively classified into the same two different groups. Like the factorial analysis, the cluster analysis opposes the operations that transform shape in its very structure and its constitution, here regrouped into the category of structural shape operators, to those that serve only to manipulate its appearance and to represent its various intermediate states, in order to evaluate and to test them, respectively encompassed by the categories of plastic and figurative shape operators. These results mean that the second level of evaluation of the model is positive, and that the shape operators of the developed model can be classified using the three categories of shape operators identified by this work.
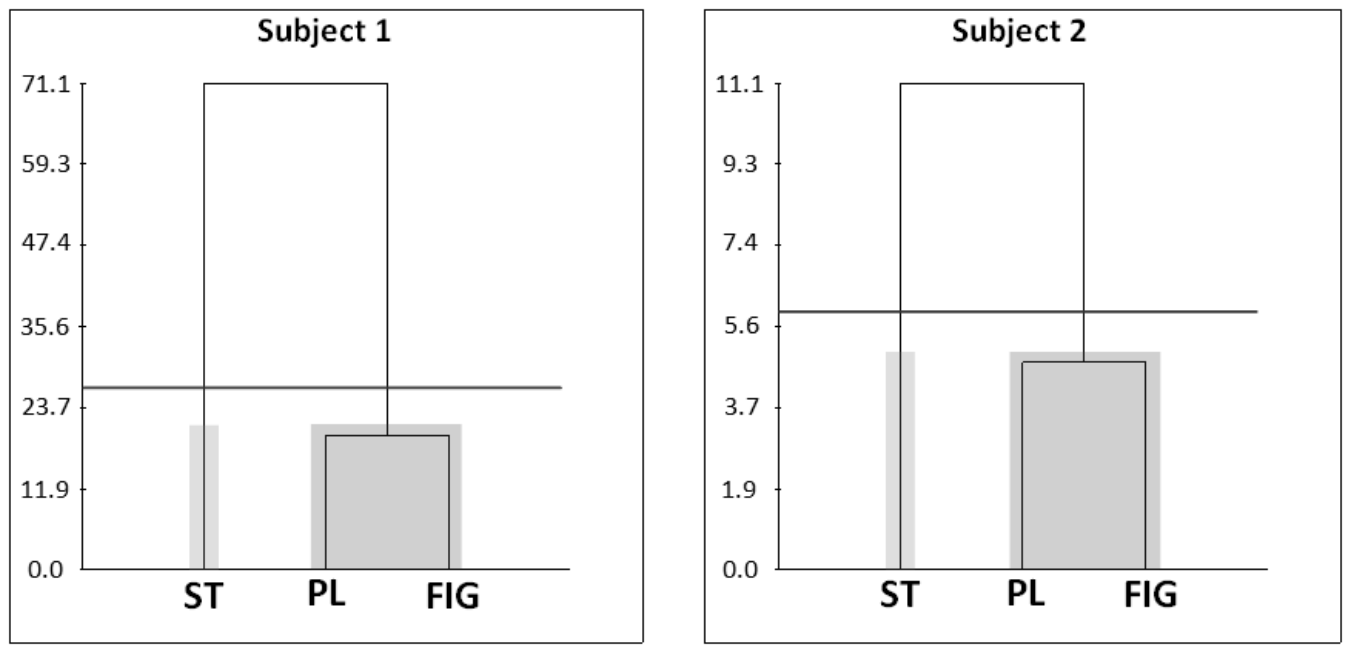

Figure 5. Results of the cluster analysis of the subjects 1 and subject 2 protocols (PL: plastic operators, ST: structural operators, FIG: figurative operators).

\section{DISCUSSION}

The present work shows that the passages from one sketch to another, during the designing activity, are made possible by a limited repertory of shape operators. They belong to three registers which allow the accomplishment of different types of design actions. Plastic operators are used to change the appearance and the contour of a shape or an element. They allow the refinement of the architectural form. Structural operators are mobilized to explore different solutions that relate to the organization, the layout and the constitution of the design. They often lead to radical changes of its shape. Finally, figurative operators are used for figuration and representation of the design object. They are mainly sought to allow the exploration, the visualization and the appraisal of the shape transformations 
undertaken by designers. Their goal is to test graphically the solutions developed by architects, through the operators of the two other registers.

\section{CONCLUSION}

This research aims to study the transformations of form, operated by architects, during the phase of form manipulation and generation of the architectural designing process. It shows that the Process of form manipulation and generation can be described by means of a model that includes a limited number of shape operators. The codification, carried out here, shows that all the operators, categories and classes of shape operators, identified in this work, are necessary for an exhaustive description of the form manipulation and generation process. Thus, our model allows to better identify the transformation of form operations, performed by designers, and to discover the logics and the mechanisms, which govern them, during the phase of form manipulation of the design process. Our findings suggest that such operators can be integrated and implemented into a computer tool intended to assist designers and architects, during the process of the creation and the development of architectural forms.

\section{REFERENCES}

Arrouf, A. and Bensaci, A: (2006), Modélisation du processus de conception, Étude expérimentale du système compositionnel, instance conception, in Courrier du savoir, Vol. 7, pp. 67-86.

Arrouf, A. (2012), Vers une théorie scientifique de la conception architecturale, contribution à l'épistémologie architecturale et à la modélisation de l'acte de concevoir, Editions Universitaires Européennes, Searbrucken, Germany.

Borie, A., Micheloni, P. and Pinon, P. (2006), Forme et déformation des objets architecturaux et urbains, Editions Parenthèses, Paris.

Ching, F.D.K. (2007), Architecture : Form, Space \& Order, Third Edition, John Wiley \& Sons, Inc., Hoboken, New Jersey.

De Biasi, P-M. (2000), pour une approche génétique de l'architecture, in Genesis, $\mathrm{n}^{\circ} 14 / 2000$, Centre canadien d'architecture et Jean Michel Place, Paris, pp.13-66.

Do, E.Y., Gross, M.D., Neiman, B. and Zimring, C. (2000), Intentions in and relations among design drawings, http://doi.org/10.1016/s0142-694x(00)00020-x

Eckersley, M., The form of design processes: a protocol analysis study (1998), http://doi.org/10.1016/0142694x(88)90034-8

Göel, V., Sketches of Thought (1995), The MIT Press, Cambridge, MA.

Jowers, I., Hogg, D. C., McKay, A., Chau, H. H. and De Pennington, A. (2010), Shape detection with vision: implementing shape grammars in conceptual design, http://doi.org/10.1007/s00163-010-0088-z

Jiang, H. And Yen, C-C. (2009), Protocol Analysis in Design Research: a review.

Lim, S., Prats, M., Jowers, I., Chase, S., Garner, S. W. and McKay, A. (2008), Shape Exploration in Design: Formalising and supporting a transformational Process. http://doi.org/10.1260/147807708787523303

Prats, M., Lim, S. and Jowers, I. (2008), "Describing shape transformations in design sketches", Design Computing and Cognition DCC'08 workshop on Informing computational support for conceptual design: lessons learned from sketching studies, 21 June 2008, Georgia Institute of Technology, Atlanta, GA, pp. 5-8.

Prats, M; Lim, S.; Jowers, I.; Garner, S. W. and Chase, S. (2009), Transforming shape in design: observations from studies of sketching. http://doi.org/10.1016/j.destud.2009.04.002

Rodgers, P. A.; Green, G. and McGowan, A. (2000), Using concept sketches to track design progress. http://doi.org/10.1016/s0142-694x(00)00018-1

Suwa, M.; Purcell, T. A. and Gero, J. S. (1998), Macroscopic analysis of design processes based on a scheme for coding designers' cognitive actions. http://doi.org/10.1016/s0142-694x(98)00016-7

Suwa, M.; Gero, J. S. and Purcell, T. A. (2006), The role of sketches in early conceptual design processes.

Terzidis, C. (1989), Transformational design.

Trescak, T., Esteva, M. and Rodriguez, I. (2012), A shape grammar interpreter for rectilinear forms. http://doi.org/10.1016/j.cad.2012.02.009

Wetzel, J. P. (2009), Proposition d'opérateurs morphosémantiques paramétriques d'assistance à la conception de formes architecturales, Phd. Thesis, École nationale supérieure d'architecture de Nancy.

Wetzel, J. P., Bignon, J-C, and Belblidia, S. (2006), "Use of morphological operators to assist architectural design in early stage". http://doi.org/10.1007/978-1-4020-5060-2_26

Yang, S. C. (2002), Reconceptualising think-aloud methodology: refining the encoding and categorizing techniques via contextualized perspectives. http://doi.org/10.1016/s0747-5632(02)00011-0 